Historic, Archive Document

Do not assume content reflects current scientific knowledge, policies, or practices. 



\title{
List of Hardy Perennial Plants
}

\author{
GROWN AND OFFERED FOR SALE BY
}

\author{
EDWARD J. CANNING,
}

11,

Prospect Heights Nursery, Northampton, Mass.

Shipping Facilities. Northampton is 100 miles east of Boston, about 140 miles north of New York and about 18 miles north of Springfield, and has excellent shipping facilities in every direction. As all my plants are field grown, they are too large to send by mail and will invariably be sent by express unless otherwise instructed. Large orders of Paeonies and other dormant roots may be safely sent by freight.

Terms. Cash from unknown correspondents or satisfactory references.

Remittances should be made by Post Office or Express Money Order, by Check, or Registered Letter. Postage stamps may be sent for amounts under $\$ 1.00$.

Prices. The prices in this list are net, except where extensive plantings would justify a special quotation.

Guarantee. I guarantee that all plants sent out shall leave here in good condition and that they shall be true to description, and if, through error any should prove untrue, to replace them without charge. It is obvious that I cannot guarantee that customers will make them live, as I have no control over conditions surrounding them and the care they will receive after they pass out of my control.

\section{LIST OF HARDY PERENNIAL PLANTS}

Commonly called old-fashioned

\section{GARDEN FLOWERS FOR FALL AND SPRING PLANTING}

Prices: Single plants 15 cts., $\$ 1.50$ per doz.; $\$ 10.00$ per 100 , ex $=$ cept where noted; 6 plants at the rate per doz., 50 plants at the rate per 100 .

ACHILLEA ptarmica var. the Pearl

ACONITUM autumnale (Monkshood)

AGROSTEMMA coronaria (Mullein Pink)

$$
\begin{array}{rrr}
\text { White } & 2 \mathrm{ft} . & \text { July-Aug. } \\
\text { Blue } & 2 \mathrm{ft} . & \text { Aug.-Sept. } \\
\text { Purple } & 4 \mathrm{ft} . & \text { June-July }
\end{array}
$$

“

$$
\text { “ var. }
$$

AQUILEGIA (Columbine)

Crimson 2-3 ft. June-Aug. White 2-3 ft. June-Aug.

"Mrs. Scott Elliott's Hybrids

، Veitch's long-spurred Hybrids

various $3 \mathrm{ft}$

June

various $3 \mathrm{ft}$

“ California Hybrids Yellow and Red $3 \mathrm{ft}$.

" vulgaris Hybrids

ARMERIA maritima (Sea Thrift)

various

Pink $1 \mathrm{ft}$.

$1 \mathrm{ft}$.

June

June

June

May-June

Purple 4-5 ft. Oct-Nov. 
ASTILBE Japonica (Japanese Astilbe) White $1 \frac{1}{2} \mathrm{ft}$. June-July " " var. compacta White $1 \mathrm{ft}$. June-July “ “ var. Queen Alexandra Pink $2 \mathrm{ft}$. June-July " " var. rubens Rose $2 \mathrm{ft}$. June-July Prices of Astilbes 20 cts. each, $\$ 2.00$ per doz.

ARABIS albida (Rock Cress) rosea

ALYSSUM saxatile compactum

AM SONIA angustifolia

BOCCONIA cordata Plume Poppy

BOLTONIA asteroides

" latisquama

CAMPANULA calycanthema

Cup and Saucer Camp

White $1 \mathrm{ft}$.
Rose $1 \mathrm{ft}$.
Yellow $1 \mathrm{ft}$.
Mauve $2 \mathrm{ft}$
Pinkish $6 \mathrm{ft}$
White $5 \mathrm{ft}$.
Lilac $5 \mathrm{ft}$.
aucer Camp

Blue $11 / 2 \mathrm{ft}$ carpatica Blue Bells var. turbinata

“

66

66

66

66

66

“

Sibirica

S'TIUM tomentosum glomerata var. dahurica

Blue

Purple

$1 \mathrm{ft}$. latifolia var. Macrantha

Purple 3-4 ft. persicifolia Peach-leaved Campanula

Blue $2-3 \mathrm{ft}$

White $2-3 \mathrm{ft}$.

pyramidalis Chimney Campanula

Blue

Violet

Yellow

White

CHRYSANTHEMUM King Edward VII White

CLEMATIS recta

White

CONVALLARIA majalis Lily of the Valley

White

$\begin{array}{ll}\text { CENTRANTHUS ruber } & \text { Red Valerian } \\ \text { var. Alba }\end{array}$

Crimson

White

Yellow

$3-4 \mathrm{ft}$.

$2 \mathrm{ft}$.

$3 \mathrm{ft}$.

$1 \mathrm{ft}$.

$2-3 \mathrm{ft}$.

$3 \mathrm{ft}$.

$1 \mathrm{ft}$.

$2-3 \mathrm{ft}$.

$2-3 \mathrm{ft}$.

May-June

May-June

May-June

May-June

August

Sept.-Oct.

Aug.-Sept.

June-July

June-July

June-July

June-July

July-Aug.

June-July

June-July

June-July

June-July

July-Aug.

May-June

July-Aug.

July-Aug.

COREOPSIS grandiflora

DELPHINIUM Larkspurs

" Brunonianum Musk Larkspur

"

formosum Hybrids

Blue, Purple margins

“

Belladonna Hybrids

chinense

Blue

6 Gentian Blue

DIANTHUS barbatus Sweet William various " " Newport Pink

“ carthusianorum Pink

Crimson

plumarius Garden Pinks

various

DICENTRA eximia Alleghany Bleeding Heart

Pink

DICTAMNUS Fraxinella Gas Plant Rosy Purple DIGITALIS purpurea Foxglove

Purple and White

ERYNGIUM planum Sea Holly

FUNKIA lancifolia Day Lily

Steel Blue

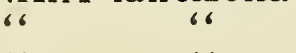

var. albo-marginata

var. undulata

" subcordata grandiflora

Blue $11 / \mathrm{ft}$

Blue $11 / 2 \mathrm{ft}$.

May-June

June-July

June-July

2 ft. Ju. -Jul. -Aug.

$1 \frac{1}{2} \mathrm{ft}$

$4-5 \mathrm{ft}$.

$3-4 \mathrm{ft}$

$2 \mathrm{ft}$.

$2 \mathrm{ft}$.

$2 \mathrm{ft}$.

$2 \mathrm{ft}$.

$1 \frac{1}{2} \mathrm{ft}$.

$11 / 2 \mathrm{ft}$

$2 \mathrm{ft}$.

$3-4 \mathrm{ft}$

3-4 $\mathrm{ft}$.

Blue $11 / 2 \mathrm{ft}$.

White

GAILLARDIA grandiflora superba Orange Red

GYPSOPHILA paniculata Baby's Breath

$1 \frac{1}{2}-2$ ft. July-Aug.

$2 \mathrm{ft}$. July-Aug.

June-July July-Aug.

July-Aug.

July-Aug.

White $2 \mathrm{ft}$.

Flore pleno White $2 \mathrm{ft}$.

HELENIUM autumnale suberbum rubrum

Yellow

Mahogony

Yellow. $2 \mathrm{ft}$.

$4 \mathrm{ft}$.

$3 \mathrm{ft}$.

$2 \mathrm{ft}$.

$3 \mathrm{ft}$.

$2 \mathrm{ft}$.

Kwanso double Orange Red

Thunbergi

HEUCHERA sanguinea

66

“

Lemon Yellow

Scarlet

$11 / 2 \mathrm{ft}$

July-Aug.

July-Aug.

Sept.-Oct.

Sept.-Oct.

June-July

June-July

July-Aug.

July-Aug.

June-July

White and Pink $3 \mathrm{ft}$

May-June 


\section{GERMAN IRIS}

IRIS florentina alba florentina blue

"Fontanrabie

" Honorabilis

“ Kharput

“ Mdme Chereau

“ Purple King

" ochroleuca

“ pseudacorus

" sibirica
Porcelain White

Yale Blue rich Blue

Yellow and Brown Blue

White, edged Blue rich Purple

Lemon Yellow clear Yellow

White and Blue
$2 \mathrm{ft}$.

$2 \mathrm{ft}$.

$2 \mathrm{ft}$.

$2 \mathrm{ft}$.

$2 \mathrm{ft}$.

$3 \mathrm{ft}$.

$2 \mathrm{ft}$.

$4 \mathrm{ft}$.

$3 \mathrm{ft}$.

$2 \mathrm{ft}$.
May-June May-June May-June May-June May-June June-July June-July July June-July June-July

Prices : 10 cts. per plant, $\$ 1.00$ per doz., $\$ 8.00$ per 100

\section{JAPANESE IRIS}

IRIS laevigatus in the following colors:

3.4 ft. July-Aug.

Pure white, rose pink, white veined blue, blue veined white, mottled blue and white, pure blue, blue with yellow throat, rich purple

Prices : 25 cts. per plant, $\$ 2.50$ per doz., $\$ 15.00$ per 100

\begin{tabular}{|c|c|c|c|}
\hline LAVANDULA vera Lavender & Blue & $2 \mathrm{ft}$. & $\begin{array}{l}\text { July-Aug } \\
\text { Mav-Ano }\end{array}$ \\
\hline LINUM perenne Flax & Blue & & May-Aug \\
\hline LOBELIA cardinalis Cardinal Flower & Crimson & $4 \mathrm{ft}$. & July-Sep \\
\hline "var. Queen Victoria & Crimson & $4 \mathrm{ft}$. & July-Sep \\
\hline LUPINUS polyphyllus roseus & Pink & $3 \mathrm{ft}$. & June-Au \\
\hline YCHNIS chalcedonica & Scarlet & $4 \mathrm{ft}$. & June-Ju \\
\hline YSIMACHIA clethroides & White & $3 \mathrm{ft}$. & July-Aus \\
\hline ONARDA didyma Bergamot & Crimson & $3 \mathrm{ft}$. & July-Aus \\
\hline MYOSOTIS palustris Forget-me-not & Blue & & June-Au \\
\hline PACHYSANDRA terminalis & White & $1 \mathrm{ft}$. & June-Au \\
\hline PAPAVER nudicaule Iceland Poppy & various & $11 / 2 \mathrm{ft}$. & May-Ju \\
\hline " orientale Oriental Poppy & Scarlet & $3 \mathrm{ft}$. & \\
\hline PENTSTEMON laevigatus & White & & Ju \\
\hline HYSOSTEGIA virginica & Lilac & $4 \mathrm{ft}$ & July-Aus \\
\hline PLATYCODON grandiflora & Blue & $3 \mathrm{ft}$. & July-Sept \\
\hline POLEMONIUM caeruleum Jacob's La & er Blue & & May-Aú \\
\hline 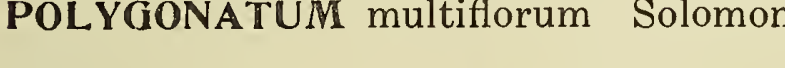 & & & \\
\hline MULA polyantha & & & \\
\hline “ vulgaris English Primro & Yellow & $1 \mathrm{f}$ & May-Jur \\
\hline YRETHRUM roseum & Pink & $2 \mathrm{f}$ & June-Aus \\
\hline
\end{tabular}

\section{THE PAEONIES}

Albatre Ivory-white, center petals tinged lilac

Boule-de-Niege Very large, white, flecked crimson

Edouard Andre Amaranth red

Festiva maxima Pure white, splashed crimson

Jean D'Arc Pink, white and sulphur

L'Eclatante Brilliant red

L'Indispensible Lilac-white, shading to rose

Mdme. Crousse White, center flecked crimson .

Mdme. Forel Silvery pink

Marie Houillon Fine large clear pink

Marie Lemoine Large pure white

Monsieur Krelage Wine red

Pottsii plena Peach color

Souv. de la Exposition Universalle Large silvery pink

Prices of the above Paeonies 50 cts. each, $\$ 5.00$ per doz. 
Canari White, light yellow center

Charlemagne Creamy white, blush center

Cytheree Flesh color, carmine spotted center

Duchess de Nemours Sulphur and white

Duke of Wellington White, sulphur center

Modeste Guerin Deep pink

Prices of the above 35 cts. each, $\$ 3.50$ per doz.

Paeony Moutan or Tree Paeonies, $\$ 1.00$ each

\section{THE PHLOXES}

Abel Tasman White with red centre

Boule de Feu Brilliant red

Cocquelicot Bright orange-red

Dr. Charcot Lavender, white centre

Eclairer Carmine red, large trusses

Elizabeth Campbell Light salmon, rose centre

Embrassement Salmon rose

Fraulein von Lassberg Pure snow white

G. A. Strohlein Scarlet orange, bright carmine center

Hanny Pfeiderer White with carmine center

Independence Pure white

Iris striped lavender and white

Jean D'Arc Pure white, very fine

Josephine Gerbeaux Pure white, rosy centre

Mdme. Paul Dutrie Soft pink

Pantheon Dark pink, large flowers

Rynstrom Beautiful clear pink

Rheingau White with violet buds

Wolfgang von Goethe Deep salmon-rose, white centre

Prices : 15 cts. each, $\$ 1.50$ per doz., $\$ 10.00$ per 100

Mixed unnamed varieties of PhIox, $\$ 1.00$ per doz.

Phlox sublata, Moss Pink, May, June $\$ 1.50$ per doz.

SALVIA officinalis

SEDUM spectabile roseum

STELLARIA Holostea Star-wort

STOKESIA cyanea Cornflower Aster

SHASTA Daisy see Chrysanthemum

THYMUS Vulgaris Thyme

TUNICA Saxifraga

ULMARIA rubra

VERONICA incana

" grandis

“ spicata “ longifolia subsessilis

VINCA minor

YUCCA filamentosa

Sage Blue $3 \mathrm{ft}$. July

Pink 11/2 ft. Aug.-Oct.

White $11 / 2 \mathrm{ft}$. May-June

Blue $3 \mathrm{ft}$. July-Aug.

Ornamental Trees and Shrubs, Evergreens and Vines in great variety and at moderate prices.

EDWARD J. CANNING,

Prospect Heights Nursery,

NORThampton, Mass. 